PROCEEDINGS OF THE

AMERICAN MATHEMATICAL SOCIETY

Volume 136, Number 9, September 2008, Pages 3255-3261

S 0002-9939(08)09066-7

Article electronically published on May 6, 2008

\title{
TOPOLOGY OF THREE-MANIFOLDS WITH POSITIVE P-SCALAR CURVATURE
}

EDWARD M. FAN

(Communicated by Richard A. Wentworth)

\begin{abstract}
Consider an $n$-dimensional smooth Riemannian manifold $\left(M^{n}, g\right)$ with a given smooth measure $m$ on it. We call such a triple $\left(M^{n}, g, m\right)$ a Riemannian measure space. Perelman introduced a variant of scalar curvature in his recent work on solving Poincaré's conjecture $P(g)=R_{\infty}^{m}(g)=$ $R(g)-2 \Delta_{g} \log \phi-|\nabla \log \phi|_{g}^{2}$, where $d m=\phi \operatorname{dvol}(g)$ and $R$ is the scalar curvature of $\left(M^{n}, g\right)$. In this note, we study the topological obstruction for the $\phi$-stable minimal submanifold with positive $P$-scalar curvature in dimension three under the setting of manifolds with density.
\end{abstract}

\section{MANifold With DENSITY}

By a Riemannian measure space we mean a triple $\left(M^{n}, g, m\right)$, where $\left(M^{n}, g\right)$ is an $n$-dimensional smooth oriented Riemannian manifold and $m$ is a smooth measure defined on $M^{n}$. Given a Riemannian manifold, there is a natural measure associated with it, i.e. the Riemannian volume measure $d v o l(g)$. By the Radon-Nikodým theorem, there exists a smooth function $\phi>0$ on $M^{n}$ such that

$$
d m=\phi d v o l(g) .
$$

Here $\phi>0$ is called the density of the manifold. The triple $\left(M^{n}, g, \phi\right)$ is called the Riemannian manifold with density $\phi$. Clearly, the study of Riemannian measure space is equivalent to the study of manifolds with density.

The study of manifolds with density traces back to the work of Bakry-Émery [1] in the early 1980s, in which they introduced the Bakry-Émery-Ricci tensor in the study of the diffusion process:

$$
R c_{\infty}^{m}(g)=R c(g)-\nabla_{g}^{2} \log \phi
$$

where $d m=\phi d \operatorname{vol}(g)$. Gromov [5] studied the manifold with density $\left(M^{n}, g, \phi\right)$ and introduced the generalized mean curvature

$$
H_{\infty}^{m}(g)=H(g)+\langle N, \nabla \log \phi\rangle,
$$

where $H$ denotes the mean curvature and $N$ is the unit normal vector field. Lott 8 studied the topological consequences of positive or nonnegative $R c_{\infty}^{m}(g)$. Morgan [9]

Received by the editors April 17, 2006, and, in revised form, November 30, 2006.

2000 Mathematics Subject Classification. Primary 53C21; Secondary 58E12, 49Q05.

Key words and phrases. Minimal submanifold, scalar curvature, Riemannian geometry.

The author was partially supported by an NSF graduate research fellowship.

(C)2008 American Mathematical Society Reverts to public domain 28 years from publication 
studied the isoperimetric inequalities of manifolds with density. In the recent work of Perelman [10] on solving Poincaré's conjecture, he introduced the new functional

$$
\mathcal{F}(g)=\int_{M} R_{\infty}^{m} d m
$$

and showed that the variation of his $\mathcal{F}$-functional is

$$
\delta \mathcal{F}\left(v_{i j}, h\right)=\int_{M}-\left\langle v, R c_{\infty}^{m}\right\rangle+\left(\frac{v}{2}-h\right)\left(R_{\infty}^{m}\right) d m
$$

where $d m=\phi d \operatorname{vol}(g)$ and $R_{\infty}^{m}(g)=R(g)-2 \Delta_{g} \log \phi-|\nabla \log \phi|_{g}^{2}$. We call $R_{\infty}^{m}(g)$ the $P$-scalar curvature. Perelman showed that $P$-scalar curvature is not the trace of the Barkry-Émery-Ricci tensor, but it relates to the Bakry-Émery-Ricci tensor under the Bianchi identity:

$$
\nabla^{* m} R c_{\infty}^{m}=\frac{1}{2} R_{\infty}^{m}
$$

where $\nabla^{* m}$ is the $L^{2}$ adjoint of $\nabla$ with respect to the measure $d m$. Both the Bakry-Émery-Ricci tensor and $P$-scalar curvature are, in some sense, the natural "infinite-dimensional" space analog of Ricci and scalar curvatures, respectively, in finite-dimensional space. More recently, Chang-Gursky-Yang [3, 4] introduced new notions for Ricci and scalar curvatures for manifolds with density $R c_{n}^{m}(g)$ and $R_{n}^{m}(g)$, respectively:

$$
R c_{n}^{m}(g)=R c(g)-\frac{2(n-1)}{n} \Delta_{g} \log \phi-\frac{(n-1)(n-2)}{n^{2}}|\nabla \log \phi|_{g}^{2}
$$

and

$$
R_{n}^{m}(g)=R(g)-\frac{2(n-1)}{n} \Delta_{g} \log \phi-\frac{(n-1)(n-2)}{n^{2}}|\nabla \log \phi|_{g}^{2},
$$

where $n$ is the dimension of $\left(M^{n}, g\right)$ and $d m=\phi d v o l(g)$. They showed that $R c_{n}^{m} \rightarrow R c_{\infty}^{m}$ and $R_{n}^{m} \rightarrow R_{\infty}^{m}$ as the dimension $n \rightarrow \infty$. (For the rigorous statement, cf. [3], [4.) These new tensors have the properties of being conformally invariant under conformal change of metrics with fixed measure $d m$.

Given a Riemannian manifold with density $\left(M^{n}, g, \phi\right)$, we consider a smooth immersed hypersurface $\Sigma^{n-1} \subset M^{n}, \partial \Sigma^{n-1}=\emptyset$. Let $F: \Sigma \times(-\epsilon, \epsilon) \rightarrow M$ be a map with compact support such that $F(x, 0)=x$ for all $x \in \Sigma$. We call such a map a variation of $\Sigma$.

Definition 1.1. An immersed submanifold $\Sigma^{n-1} \subset M^{n}$ is called $\phi$-minimal if

$$
\left.\frac{d}{d t}\right|_{t=0} \operatorname{Vol}_{\phi}(F(\Sigma, t))=\left.\frac{d}{d t}\right|_{t=0} \int_{\Sigma} F^{*} \phi d \operatorname{vol}\left(F^{*} g\right)=0 \text { for all variations } F \text {. }
$$

Definition 1.2. An immersed submanifold $\Sigma^{n-1} \subset M^{n}$ is called $\phi$-stable minimal if it is $\phi$-minimal and

for all variations $F$.

$$
\left.\frac{d^{2}}{d t^{2}}\right|_{t=0} \operatorname{Vol}_{\phi}(F(\Sigma, t)) \geq 0
$$

Bayle 2] derived the first and second variation formulae for the weighted volume functional (cf. 13] for a detailed presentation of such derivation). From the first variation formula, it's easy to see that an immersed submanifold $\Sigma^{n-1} \subset M^{n}$ is $\phi$-minimal if and only if the generalized mean curvature $H_{\phi}=H(g)+\langle N, \nabla \log (\phi)\rangle$ $=0$, where $H$ is the mean curvature with respect to a unit normal $N$ to $\Sigma$. From the second variation formula, it is also straightforward to obtain the following lemma. 
Lemma 1.3. For a $\phi$-stable minimal submanifold $s: \Sigma^{n-1} \rightarrow\left(M^{n}, g, m\right), d m=$ $\phi \operatorname{dvol}(g)$, the following inequality holds for any smooth function $\eta$ with compact support in $\Sigma^{n-1}$ :

$$
\int_{\Sigma} R c_{\infty}^{m}(N, N) \eta^{2} s^{*}(d m)+\int_{\Sigma}|S|^{2} \eta^{2} s^{*}(d m) \leq \int_{\Sigma}\left|\nabla_{\Sigma} \eta\right|^{2} s^{*}(d m),
$$

where $N$ is the unit normal vector field on $\Sigma^{n-1}, S$ is the shape operator on $\Sigma$ and $R c_{\infty}^{m}(g)=R c(g)-\nabla^{2} \log \phi$ is the Bakry-Émery-Ricci curvature.

Corollary 1.4. Suppose $(M, g, \phi)$ has Bakry-Émery-Ricci curvature $R c_{\infty}^{m}>0$. Then there exist no compact $\phi$-stable minimal immersed submanifolds of codimension one.

Proof. Take $\eta=1$ in Lemma 1.3. Then the conclusion follows.

Remark 1.5. In the case $\phi=$ constant, this reduces to the well-known result that $\left(M^{n}, g\right)$ with positive Ricci curvature has no compact immersed stable minimal submanifolds of co-dimension 1.

\section{Main Results}

In this section, we generalize two results of Schoen and Yau on stable minimal submanifolds and characterize totally geodesic submanifold of codimension one, all in the setting of manifolds with density.

Schoen and Yau [15] proved that if $\left(M^{3}, g\right)$ is oriented and has positive scalar curvature, then it has no compact, immersed stable minimal surfaces of positive genus. We can generalize this result as follows.

Theorem 2.1. Let $\left(M^{3}, g, m\right)$ be a smooth oriented three-manifold with measure $d m=\phi d v o l(g)$. If $R_{\infty}^{m}>0$, then there is no compact immersed $\phi$-stable minimal two-dimensional submanifold with positive genus.

Proof. Let $\Sigma^{2} \subset M^{3}$ be a compact immersed $\phi$-stable minimal submanifold. Taking $\eta=\phi^{-\frac{1}{2}}$ in Lemma 1.3, we get

$$
\int_{\Sigma} R c_{\infty}^{m}(N, N) \operatorname{dvol}\left(\left.g\right|_{\Sigma}\right)+\int_{\Sigma}|S|^{2} d v o l\left(\left.g\right|_{\Sigma}\right) \leq \frac{1}{4} \int_{\Sigma}\left|\nabla_{\Sigma} \log \phi\right|^{2} d v o l\left(\left.g\right|_{\Sigma}\right) .
$$

Let $K$ denote the Gauss curvature of $\Sigma^{2}$, and take an orthonormal frame $e_{i}$ with respect to $\left(M^{3}, g\right)$, where the index $i=1,2$ denotes the vector fields which are tangential to $\Sigma^{2}$ and $i=3$ denotes the vector field in the normal direction. Let

$$
h_{i j}=S\left(e_{i}, e_{j}\right)=\left\langle\nabla_{e_{i}} N, e_{j}\right\rangle \quad(i=1,2)
$$

and let $R_{i j}$ denote the sectional curvature of $e_{i} \wedge e_{j}$ and $R=\operatorname{tr}(R c)$ the scalar curvature. As in Schoen-Yau [15], we have

$$
\begin{aligned}
R c(N, N)+|S|^{2} & =R_{13}+R_{23}+\Sigma_{i, j=1}^{2} h_{i j}^{2} \\
& =\frac{1}{2} R-K+h_{11} h_{22}+h_{12}^{2}+h_{11}^{2}+h_{22}^{2} \\
& =\frac{1}{2} R-K+\frac{1}{2}\left(h_{11}+h_{22}\right)^{2}+\frac{1}{2} \Sigma_{i=1}^{2} h_{i j}^{2}
\end{aligned}
$$


and therefore it follows from (2.1) that

$$
\begin{aligned}
& \int_{\Sigma}\left(\frac{1}{2} R\right.\left.-\left\langle\nabla_{N} \nabla \log \phi, N\right\rangle+\frac{1}{2} H^{2}+\frac{1}{2}|S|^{2}\right) \operatorname{dvol}\left(\left.g\right|_{\Sigma}\right) \\
& \leq \int_{\Sigma} K \operatorname{dvol}\left(g_{\Sigma}\right)+\frac{1}{4} \int_{\Sigma}\left|\nabla_{\Sigma} \log \phi\right|^{2} d \operatorname{vol}\left(\left.g\right|_{\Sigma}\right) .
\end{aligned}
$$

On the other hand, apply the divergence theorem:

$$
\begin{aligned}
& \int_{\Sigma} \Delta_{g} \log \phi \operatorname{dvol}\left(\left.g\right|_{\Sigma}\right) \\
& =\int_{\Sigma} \Sigma_{i=1}^{2}\left(\left\langle\nabla_{e_{i}}(\nabla \log \phi)^{T}, e_{i}\right\rangle+\left\langle\nabla_{e_{i}}(\langle\nabla \log \phi, N\rangle N), e_{i}\right\rangle\right) \operatorname{dvol}\left(\left.g\right|_{\Sigma}\right) \\
& +\int_{\Sigma}\left\langle\nabla_{N} \nabla \log \phi, N\right\rangle d \operatorname{vol}\left(\left.g\right|_{\Sigma}\right) \\
& =\int_{\Sigma} \operatorname{div}_{\Sigma}(\nabla \log \phi)^{T} d \operatorname{vol}\left(\left.g\right|_{\Sigma}\right)+\int_{\Sigma}\left(\langle\nabla \log \phi, N\rangle H+\left\langle\nabla_{N} \nabla \log \phi, N\right\rangle\right) \operatorname{dvol}\left(\left.g\right|_{\Sigma}\right) \\
& =\int_{\Sigma}\left(\langle\nabla \log \phi, N\rangle H+\left\langle\nabla_{N} \nabla \log \phi, N\right\rangle\right) \operatorname{dvol}\left(\left.g\right|_{\Sigma}\right) .
\end{aligned}
$$

Combining the above calculations, we obtain

$$
\begin{aligned}
& \frac{1}{2} \int_{\Sigma} R_{\infty}^{m} \operatorname{dvol}\left(\left.g\right|_{\Sigma}\right) \\
& =\frac{1}{2} \int_{\Sigma} R d \operatorname{dvol}\left(\left.g\right|_{\Sigma}\right)-\int_{\Sigma} \Delta_{g} \log \phi d v o l\left(\left.g\right|_{\Sigma}\right)-\frac{1}{2} \int_{\Sigma}|\nabla \log \phi|^{2} d v o l\left(\left.g\right|_{\Sigma}\right) \\
& =\int_{\Sigma}\left(\frac{1}{2} R-\left\langle\nabla_{N} \nabla \log \phi, N\right\rangle+\frac{1}{2} H^{2}+\frac{1}{2}|S|^{2}-\frac{1}{4}\left|\nabla_{\Sigma} \log \phi\right|^{2}\right) d v o l\left(\left.g\right|_{\Sigma}\right) \\
& \left.+\int_{\Sigma}-\frac{1}{2} H^{2}-\frac{1}{2}|S|^{2}-\langle\nabla \log \phi, N\rangle H-\frac{1}{2}|\nabla \log \phi|^{2}+\frac{1}{4}\left|\nabla_{\Sigma} \log \phi\right|^{2}\right) d v o l\left(\left.g\right|_{\Sigma}\right) \\
& \leq \int_{\Sigma} K \operatorname{dvol}\left(\left.g\right|_{\Sigma}\right)-\frac{1}{2} \int_{\Sigma}\left(H_{\phi}^{2}+|S|^{2}+\frac{1}{2}\left|\nabla_{\Sigma} \log \phi\right|^{2}\right) d v o l\left(\left.g\right|_{\Sigma}\right) \\
& =\int_{\Sigma} K \operatorname{Kvol}\left(\left.g\right|_{\Sigma}\right)-\frac{1}{2} \int_{\Sigma}\left(|S|^{2}+\frac{1}{2}\left|\nabla_{\Sigma} \log \phi\right|^{2}\right) \operatorname{dvol}\left(\left.g\right|_{\Sigma}\right) \\
& \leq \int_{\Sigma} K \operatorname{Kdvol}\left(\left.g\right|_{\Sigma}\right) .
\end{aligned}
$$

By the Gauss-Bonnet theorem,

$$
2 \pi(2-2 g)=\int_{\Sigma} \operatorname{Kdvol}\left(\left.g\right|_{\Sigma}\right), \quad \text { where } g \text { is the genus of the surface } \Sigma .
$$

Hence

$$
\frac{1}{2} \int_{\Sigma} R_{\infty}^{m} \operatorname{dvol}\left(\left.g\right|_{\Sigma}\right) \leq 2 \pi(2-2 g) .
$$

The conclusion follows.

Remark 2.2. In dimension three, we can replace Perelman's $P$-scalar curvature $R_{\infty}^{m}$ with the CGY-scalar curvature $R_{n}^{m}$ in the above theorem and the conclusion still holds (cf. [3]).

We recall the definition of harmonic maps on surfaces. Let $e_{i}$ be an orthonormal frame on $\left(\Sigma^{2}, h\right)$, and let $e(s)=\sum_{i=1}^{2}\left\langle d s\left(e_{i}\right), d s\left(e_{i}\right)\right\rangle_{g}$. 
Definition 2.3. A smooth map $s:\left(\Sigma^{2}, h\right) \rightarrow\left(M^{3}, g\right)$ is harmonic if for any 1-parameter family of maps $u:\left(\Sigma^{2}, h\right) \times(-\epsilon, \epsilon) \rightarrow\left(M^{3}, g\right)$ with $u(x, 0)=s(x)$, we have

$$
\left.\frac{d}{d t} E(u(t))\right|_{t=0}=\left.\frac{d}{d t}\right|_{t=0} \int_{\Sigma} e(u(t)) \operatorname{dvol}(h)=0 .
$$

A harmonic map on a surface is a nice object to study because of its complex structure. We can complexify the tangent bundle of $M^{3}, T^{\mathbb{C}}(M)=T(M) \otimes \mathbb{C}$. We have

$$
\begin{aligned}
\partial_{z} s & =\frac{1}{2}\left(\partial_{x} s-i \partial_{y} s\right), \\
\partial_{\bar{z}} s & =\frac{1}{2}\left(\partial_{x} s+i \partial_{y} s\right) .
\end{aligned}
$$

The map $s$ is called weakly conformal if its quadratic differential

$$
\left(s^{*} g\right)^{(2,0)}=\left\langle\partial_{z} s, \partial_{z} s\right\rangle_{g}^{\mathbb{C}}=0 .
$$

Gulliver-Osserman-Royden 7 proved that if $s$ is harmonic and weakly conformal, then it is a branched immersion. It is well-known that in dimension three a branched minimal immersion is in fact immersion (cf. 6]). Based on this important observation which enables one to link harmonic maps with immersions, Sacks-Uhlenbeck 14 and Schoen-Yau [15] prove that under some non-degeneracy conditions on a map $u: \Sigma^{2} \rightarrow M^{3}$, there exists a minimal harmonic map $s$ homotopic to $u$. SchoenYau further used this result to show two important existence theorems of minimal immersions for three-manifolds. We state their results below.

Theorem 2.4 (Theorem 3.1 of [15]). Let $\Sigma^{2}$ denote a compact surface of genus $g>1$ and let $\left(M^{3}, \widetilde{g}\right)$ denote a compact Riemannian manifold. Suppose $u: \Sigma^{2} \rightarrow$ $\left(M^{3}, \tilde{g}\right)$ is a map which is injective on fundamental groups. Then there exist a conformal structure on $\Sigma^{2}$ and an immersion $s: \Sigma^{2} \rightarrow\left(M^{3}, \tilde{g}\right)$ whose action on $\pi_{1}$ is conjugate to that of $u$ and which minimizes the area over all such maps.

Theorem 2.5 (Theorem 4.1 of [15]). Let $T^{2}$ denote the two-dimensional torus and let $\left(M^{3}, \tilde{g}\right)$ denote a compact Riemannian manifold. Suppose $\pi_{1}(M)$ contains a finitely generated non-cyclic abelian group. Then there exists a minimal immersion $s: T^{2} \rightarrow\left(M^{3}, \tilde{g}\right)$ whose induced map on $\pi_{1}$ has rank 2 image in $\pi_{1}(M)$. Moreover, $s$ minimizes the area over all maps $u: T^{2} \rightarrow\left(M^{3}, \tilde{g}\right)$ which satisfy $u_{\sharp}=\tau^{-1} s_{\sharp} \tau$ : $\pi_{1}\left(T^{2}, p\right) \rightarrow \pi_{1}(M, u(p))$ for some curve $\tau$ from $u(p)$ to $s(p)$.

We can generalize Schoen-Yau's [15] work on the topological constraints of the existence of positive scalar curvature on three-manifolds as follows.

Theorem 2.6. Let $M^{3}$ be a compact, oriented, smooth manifold. Suppose either of the following two conditions holds for $M^{3}$ :

(i) $\pi_{1}(M)$ contains a finitely generated non-cyclic abelian subgroup or

(ii) $\pi_{1}(M)$ contains a subgroup abstractly isomorphic to the fundamental group of a surface of genus $g>1$.

Then $M^{3}$ admits no metrics of positive P-scalar curvature w.r.t. any density function; i.e. $M^{3}$ can have no metrics $g$ with $R_{\infty}(g, \phi)=R(g)-2 \Delta_{g} \log \phi-|\nabla \log \phi|_{g}^{2}>0$ for any smooth positive function $\phi$.

Proof. We closely follow the proof of Theorem 5.2 in Schoen-Yau ([15, p. 139). 
In our setting, $\left(M^{3}, g, \phi\right)$, we let $\tilde{g}=\phi g$. Under the hypotheses of Theorem 2.6, we can easily construct the map $s: \Sigma^{2} \rightarrow\left(M^{3}, \tilde{g}\right)$ with genus of $\Sigma^{2}$ being positive. We apply Theorem 2.4 or Theorem 2.5. In either case, the map $s$ satisfies

$$
\left.\frac{d}{d t} \operatorname{vol}(F(\Sigma, t))\right|_{t=0}=\left.\frac{d}{d t}\right|_{t=0} \int_{\Sigma} \operatorname{dvol}\left(F^{*} \tilde{g}\right)=0,
$$

for all variations $F: \Sigma \times(-\epsilon, \epsilon) \rightarrow\left(M^{3}, \tilde{g}\right), F(x, 0)=s$. Since $\Sigma^{2}$ is of dimension two,

$$
\int_{\Sigma} d \operatorname{vol}\left(F^{*} \tilde{g}\right)=\int_{\Sigma} d \operatorname{vol}\left(F^{*}(\phi g)\right)=\int_{\Sigma} \phi(F) d \operatorname{vol}\left(F^{*} g\right)=\operatorname{vol}_{\phi}(F(\Sigma, t)) .
$$

This implies that $s$ is a $\phi$-stable minimal immersion from $\Sigma^{2}$ to $\left(M^{3}, g\right)$. If $M^{3}$ has a metric $g$ such that $R_{\infty}^{m}(g)>0$, applying Theorem 2.1, we know that the map $s$ we constructed above cannot exist. This is a contradiction and completes the proof.

Remark 2.7. The $P$-scalar curvature is the limit of the scalar curvature of ChangGursky-Yang [3], $R_{n}^{m}(g) \rightarrow R_{\infty}^{m}$, as the dimension $n \rightarrow \infty$. (See Chang-GurskyYang for the precise statement.) Even though there exists a "canonical metric" $g_{n} \in[g]$ such that $R_{n}^{m}(g)=R\left(g_{n}\right)$, this is not true for the $P$-scalar curvature in general. So the topological constraints on fundamental groups indeed give a more restrictive class of metrics which can be put on $M^{3}$, thus generalizing the result of Schoen and Yau.

The Bonnet-Myers Theorem states that a complete Riemannian manifold $\left(M^{n}, g\right)$ with Ricci curvature $R c(g) \geq \lambda>0$ is compact (cf. [11]). On the other hand, Morgan ( 9 , Corollary 4) showed that any complete smooth Riemannian manifold with density $\left(M^{n}, g, \phi\right)$ with $R c_{\infty}^{m}(g) \geq \lambda>0$ must have finite weighted volume even though it is not necessarily compact. A simple example of such a case is the Gauss space. If $\Sigma^{n-1} \subset M^{n}$ is non-compact, utilizing Lemma 1.3 and Morgan (9], Corollary 4), we have the following characterization of totally geodesic submanifolds.

Theorem 2.8. Suppose $\Sigma^{n-1} \subset\left(M^{n}, g, \phi\right)$ is a complete non-compact $\phi$-stable minimal hypersurface, and let $\operatorname{Rc}_{\infty}^{m}\left(M^{n}, g, \phi\right) \geq \lambda>0$. Then $\Sigma^{n-1} \subset M^{n}$ is totally geodesic if and only if $\operatorname{vol}_{\phi}\left(\Sigma,\left.g\right|_{\Sigma}\right)=\int_{\Sigma} \phi \operatorname{dvol}\left(\left.g\right|_{\Sigma}\right)<\infty$.

Proof. Take a ball $B_{r}(x) \subset \Sigma$. Let $\eta$ be a cutoff function such that it is equal to 1 in $B_{r}(x)$ and vanishes outside $B_{3 r}(x)$ and $|\nabla \eta|<\frac{1}{r}$. Lemma 1.3 implies

$$
\int_{\Sigma} R c_{\infty}^{m}(N, N) \eta^{2} v_{0}+\int_{\Sigma}|S|^{2} \eta^{2} v_{0} \leq \int_{\Sigma}\left|\nabla_{\Sigma} \eta\right|^{2} v_{0}
$$

where $v_{0}=\phi d v o l\left(\left.g\right|_{\Sigma}\right)$. By assumption, $R c_{\infty}^{m}\left(M^{n}, g, \phi\right)>0$; therefore

$$
\int_{B_{r}(x)}|S|^{2} v_{0} \leq \int_{\Sigma} \frac{1}{r^{2}} v_{0}=\frac{v o l_{\phi}(\Sigma)}{r^{2}} \rightarrow 0, \quad \text { as } r \rightarrow \infty,
$$

provided $\operatorname{vol}_{\phi}(\Sigma)<\infty$. This implies

$$
|S| \equiv 0 \quad \text { on } \Sigma,
$$

which means that $\Sigma^{n-1} \subset M^{n}$ is totally geodesic. Conversely, if $R c_{\infty}^{m}(M, g, \phi) \geq$ $\lambda>0$ and the immersion is totally geodesic, then $R c_{\infty}^{m}\left(\Sigma,\left.g\right|_{\Sigma},\left.\phi\right|_{\Sigma}\right) \geq \lambda^{\prime}>0$. By Corollary 4 of Morgan [9, $\operatorname{vol}_{\phi} \Sigma<\infty$. 


\section{ACKNOWLEDGMENTS}

The author would like to thank Professor Paul Yang and his advisor, Professor Alice Chang, for helpful discussions. The author also thanks the referee for suggestions on improving the presentation of this paper and for pointing out the work of V. Bayle on variation formulae.

\section{REFERENCES}

[1] D. Bakry and M. Émery. Diffusions Hypercontractives, in: Séminaire de probabilités XIX, 1983/84, 177-206, Lecture Notes in Math. 1123, Springer, Berlin, 1985. MR889476 (88j:60131)

[2] V. Bayle, Propriétés de concavité du profil isopérimétrique et applications. Ph.D. Thesis.

[3] S.Y.A. Chang, M. Gursky, and P. Yang. Conformal invariants associated to a measure. Proc. Natl. Acad. Sci. USA 103 (2006), no. 8, 2535-2540. MR.2203156 (2007e:53032)

[4] S.Y.A. Chang, M. Gursky, and P. Yang. Conformal invariants associated to a measure, I: Pointwise invariants. (Preprint)

[5] M. Gromov. Isoperimetry of waists and concentration of maps. Geom. Funct. Anal. 13 (2003), no. 1, 178-215. MR $1978494(2004 \mathrm{~m}: 53073)$

[6] R.D. Gulliver II. Regularity of minimizing surfaces of prescribed mean curvature. Ann. of Math. (2) 97 (1973), 275-305. MR0317188 (47:5736)

[7] R.D. Gulliver II, R. Osserman, and H.L. Royden. A theory of branched immersions of surfaces. Amer. J. Math. 95 (1973), 750-812. MR0362153(50:14595)

[8] J. Lott. Some geometric properties of the Bakry-Émery-Ricci tensor. Comment. Math. Helv. 78 (2003), no. 4, 865-883. MR2016700 (2004i:53044)

[9] F. Morgan. Manifolds with density. Notices Amer. Math. Soc. 52 (2005), no. 8, 853-858. MR:2161354 (2006g:53044)

[10] G. Perelman. The entropy formula for the Ricci flow and its geometric applications, http://arXiv.org/abs/math.DG/0211159.

[11] P. Petersen. Riemannian Geometry, Graduate Texts in Mathematics, Springer-Verlag, New York, 1998. MR1480173 (98m:53001)

[12] A. Ros. The isoperimetric problem, Global Theory of Minimal Surfaces, Amer. Math. Soc., Providence, RI, 2005, 175-209. MR2167260 (2006e:53023)

[13] C. Ros., A. Cañete, V. Bayle, F. Morgan, On the isoperimetric problem in Euclidean space with density, http://arXiv.org/abs/math.DG/0602135.

[14] J. Sacks and K. Uhlenbeck. The existence of minimal immersions of 2-spheres. Ann. of Math. (2) 113 (1981), no. 1, 1-24. MR604040 (82f:58035)

[15] R. Schoen and S.T. Yau. Existence of incompressible minimal surfaces and the topology of three-dimensional manifolds with nonnegative scalar curvature. Ann. of Math. (2) 110 (1979), no. $1,127-142$. MR.541332 (81k:58029)

Department of Mathematics, Princeton University, Princeton, New Jersey 085441000

E-mail address: efan@math.princeton.edu 